\title{
Líneas teóricas fundamentales para una educación emocional*
}

\section{Hernando Barrios-Tao}

https://orcid.org/0000-0002-8999-0586 Universidad Militar Nueva Granada,

Colombia

hernando.barrios@unimilitar.edu.co

\section{Lina Johanna Peña Rodríguez}

https://orcid.org/0000-0002-2026-5925 Universidad Militar Nueva Granada, Colombia

lina.pena@unimilitar.edu.co

\section{Resumen}

El objetivo del artículo es trazar líneas teóricas que puedan fundamentar procesos de educación emocional. El método se inscribe en la revisión narrativa, caracterizada por investigar un tópico de forma más o menos exhaustiva, y se ubica en el espectro amplio de la relación entre emociones y educación. Los resultados, presentados en forma narrativa, inician con la comprensión de la complejidad y multidimensionalidad de las experiencias emocionales, con base en la relación entre neurociencias, emociones y procesos educativos. La conclusión fundamental es la necesidad de una educación emocional que transite de la inteligencia y las competencias socioemocionales hacia una visión ampliada al desarrollo de las experiencias emocionales, con una visión de la educación extendida a lo largo de toda la vida y a la vida misma.

\section{Palabras clave (Fuente: tesauro de la Unesco)}

Desarrollo afectivo; desarrollo emocional; educación emocional; neuroeducación; revisión narrativa; teoría de la educación.

* El artículo se asocia con el Proyecto Investigación INV-HUM-2606: "Neurociencias, educación y emociones. Fase 1: Revisión narrativa”, financiado por la Vicerrectoría de Investigaciones de la Universidad Militar Nueva Granada, Bogotá, Colombia.

Recepción: 13/11/2018 | Envío a pares: 24/04/2019 | Aceptación por pares: 21/10/2019 | Aprobación: 21/10/2019 


\title{
Fundamental Theoretical Lines for an Emotional Education*
}

\begin{abstract}
This article aims at drawing theoretical lines to support emotional education. The method used is a narrative review, characterized by a fairly thorough research into a topic, and is situated in the broad spectrum of the education-emotions relationship. Results, presented in a narrative manner, provide an understanding of the complex and multidimensional nature of emotional experiences based on the relationship among neurosciences, emotions, and educational processes. It is concluded that the$r e$ is a need for an emotional education that moves from intelligence and socio-emotional skills to the development of emotional experiences, with a vision of education that extends throughout life and to life itself.
\end{abstract}

\section{Keywords (Source: Unesco Thesaurus)}

Emotional education; emotional development; neuroeducation; narrative review; Educational theory.

\footnotetext{
* This article is associated with the Research Project INV-HUM-2606: "Neurosciences, education and emotions. Phase 1: Narrative review", funded by the Office of the Vice President for Research, Universidad Militar Nueva Granada, Bogotá, Colombia.
} 


\section{Linhas teóricas fundamentais para uma educação emocional*}

Resumo

O objetivo deste artigo é estabelecer linhas teóricas que possam fundamentar processos de educação emocional. O método se baseia numa revisão narrativa, caracterizada por pesquisar um tópico de forma mais ou menos exaustiva, e se encontra num conjunto amplo da relação entre emoções e educação. Os resultados, apresentados em forma narrativa, iniciam com a compreensão da complexidade e multidimensionalidade das experiências emocionais, com base na relação entre neurociências, emoções e processos educativos. A conclusão fundamental é a necessidade de uma educação emocional que transite da inteligência e das competências socioemocionais para uma visão ampliada do desenvolvimento das experiências emocionais, com uma perspectiva da educação estendida ao longo de toda a vida e à vida em si.

\section{Palavras-chave (Fonte: tesauro da Unesco)}

Desenvolvimento afetivo; desenvolvimento emocional; educação emocional; neuroeducação; revisão narrativa; teoria da educação.

* Este artigo está vinculado ao projeto de pesquisa INV-HUM-2606: "Neurociencias, educación y emociones. Fase 1: Revisión narrativa" ("Neurociências, educação e emoções. Fase 1: revisão narrativa"), financiado pela Vice-reitoria de Pesquisas da Universidad Militar Nueva Granada, Bogotá, Colômbia. 
En escenarios empresariales y educativos, las habilidades y competencias socioemocionales se configuran como valiosa mercancía, cuyos resultados se transforman en productividad, salud, y éxito (Mayo, 1977; Illou, 2007). Este "capital emocional" (Illou, 2007) adquiere relevancia por su necesidad en ambientes digitales que develan y exteriorizan deficiencias emocionales en las relaciones interpersonales, allí ampliadas y reconfiguradas, que limitan con patologías de ansiedad, depresión, violencia (García, 2012). El denominado "analfabetismo emocional" (Dueñas, 2002), con efectos visibles en los ámbitos personal, educativo, político, cultural y social (Bisquerra y Pérez, 2012), valora aún más la necesidad de una educación emocional.

Inteligencia emocional, competencias socioemocionales y formación emocional reflejan experiencias, prácticas y procesos importados al ámbito educativo que se vinculan con aspectos de rendimiento, bienestar y mejoramiento en los procesos de enseñanza-aprendizaje. La innegable y ya inevitable relación entre emociones y educación se percibe en resultados investigativos con algunos interrogantes todavía en vías de resolución: el papel de las emociones en aprender a enseñar, la relación de las experiencias emocionales de los maestros con sus prácticas de enseñanza, la interacción del contexto sociocultural de la enseñanza con las emociones de los maestros, la relación entre emociones y motivación, la ponderación de la experiencias emocionales integrales en el desarrollo docente (Sutton y Wheatley, 2003; Pekrun, Goetz, Titz y Perry, 2002; Pekrun, 2005). Los estudios neurocientíficos impulsados por el proyecto Década del Cerebro evidencian la relación entre emociones y procesos educativos iniciada en la antigua Grecia y desarrollan el intercambio multifacético del aprendizaje: elementos cognitivos, emocionales y fisiológicos, así como las relaciones entre procesos educativos y aspectos vinculados con el entorno sociocultural (OCDE, 2007; De Jong, 2008; Barrios-Tao, 2016).
A finales del siglo pasado Hargreaves (1998) reclamaba a quienes eran responsables de gestionar reformas educativas su ignorancia y subestimación de la "dimensión emocional" como uno de los aspectos fundamentales, no solo de procesos de enseñanza-aprendizaje, sino también de la comprensión de la vida misma de los profesores. Por otra parte, la problemática de la educación centrada en aspectos cognitivos y de competencias relacionadas con el hacer, en un escenario de mercado, conduce al descuido de habilidades del ser y convivir y de las emociones como un objetivo fundamental de la educación (Bisquerra, 2003).

La ampliación de la psicología al ámbito de la salud mental desempeñó un papel protagónico en establecer soluciones terapéuticas a los desórdenes emocionales que impiden el éxito y la autorrealización, en la búsqueda de una indefinida salud, quebrantada por conductas neuróticas, desadaptadas o disfuncionales (González, 2010). El paso de esta esfera privada a los escenarios públicos permite el arribo de los instrumentos, parámetros y procedimientos de la psicología a la educación para diagnosticar, cuantificar y evaluar inteligencia emocional y competencias socioemocionales (Mayer y Salovey, 1997; Mayer, Salovey y Caruso, 2000; Bisquerra, 2003), con base en estándares sobre personas competentes emocionalmente, con mayor posibilidad de éxito, rendimiento y productividad en los ámbitos laborales y educativos. Sin embargo, la extrapolación de los escenarios de aula en los que se desarrolla el acto educativo y la falacia del desarrollo de habilidades concluidas, en detrimento de una concepción de la educación como permanente formación del ser humano, entre otros aspectos, plantean críticas a una educación emocional que arriesga reducirse a prácticas terapéuticas y a la promoción de factores de competitividad que no contribuyen con un desarrollo integral y permanente del ser humano en constante proceso formativo (Prieto, 2018; Nobile, 2017; Marina, 2005). 
Frente a la abundancia investigativa de la psicología en diagnóstico, terapia, teorías y estrategias para la comprensión, práctica y regulación de las emociones, con exclusión de los escenarios educativos en unos casos e inclusión en otros, los estudios e investigaciones realizadas por educadores para la intervención práctica de la expresiones y experiencias emocionales en los escenarios educativos se encuentran todavía en proceso de crecimiento. Los desarrollos de las neurociencias, las investigaciones teóricas y las experiencias de los mismos educadores permiten recoger elementos para una revisión encaminada a determinar líneas teóricas que podrían fundamentar procesos de educación emocional, objetivo fundamental del artículo.

\section{Metodología}

El artículo es de revisión narrativa y se caracteriza por investigar un tópico de forma más o menos exhaustiva (Letelier, Manríquez y Rada, 2005). En este sentido la revisión es selectiva y permite desarrollar una descripción de los resultados de estudios incluidos, aspectos clave, además de comparar y contrastar las relaciones encontradas en los documentos encontrados (Popay et al.,2006). El tópico de revisión se ubica en el espectro amplio de la relación entre emociones y educación, y se determina de manera particular con la pregunta selectiva que orientó la búsqueda: ¿Qué líneas teóricas podrían fundamentar una educación emocional? La revisión se delimita por la pregunta orientadora y se enfoca en resultados investigativos teóricos, de revisión, diagnóstico e intervención de procesos educativos realizados por educadores en escenarios educativos, en bases de datos y sistemas internacionales (Ebsco, Proquest, Science Direct, Scopus). Las categorías de revisión para la búsqueda fueron: emociones, educación, profesores, estudiantes, neurociencias.

\section{Resultados y discusión}

De acuerdo con la identidad de la revisión, los resultados sobre algunas líneas teóricas que po- drían fundamentar procesos de educación emocional se presentan de forma narrativa, con base en la previa organización y análisis de los documentos examinados, realizadas por los investigadores. La discusión se centra en su aplicación en escenarios educativos y sus desarrollos, horizontes y desafíos para actores y prácticas educativas.

\section{Neurociencias, emociones y procesos educativos}

La primera línea teórica para fundamentar una educación emocional se origina en la relación emociones-educación, ya presente en los antiguos griegos y ahora impulsada por investigaciones neurocientíficas que no solo evidenciaron este vínculo entre emociones y procesos educativos (Blakemore y Frith, 2000; Goswami, 2006; Hall, 2005; Ainley, 2006; De Jong, 2008; Fontinha et al., 2009; Goetz, Cronjaeger, Frenzel, Ludtke y Hall, 2010; Yiend, 2010; Jankowski y Takahashi, 2014; Martin y Ochsner, 2016), sino que fundamentaron el hecho de que en toda práctica educativa se entrelazan e involucran tanto la dimensión racional-cognitiva como la emocionalafectiva, con la influencia de aspectos individuales, biológicos, psicológicos, cognitivos, y socioculturales (Damasio, 1996; Le Doux, 1996; Vázquez y Manassero, 2007a/b; OCDE, 2007; Fried, 2011).

Los aportes de las neurociencias a la educación y el impacto en la mejor comprensión de sus procesos se enmarcan en diferentes aspectos: relación con el entorno sociocultural, plasticidad cerebral, neuronas espejo, neuromitos, ejercicio físico, entre otros (Goswami, 2004; Blakemore y Frith, 2005; Hall, 2005; Hillman, Erickson y Kramer, 2008; Citri y Malenka, 2008; García, 2008; De Jong, 2008; Coll, 2011; Gruart, 2014). Detractores y defensores de la relación entre neurociencias y educación (Barrios-Tao, 2016) ya comenzaron a ver resultados sobre la semántica y sintaxis cerebral ligadas con procesos educativos (Bisquerra, 2003; OCDE, 2007; De Jong, 2008; Marina, 2012) y asimismo se cuenta con investigaciones 
realizadas por educadores acerca del impacto de las emociones en escenarios y prácticas de enseñanzaaprendizaje, formación, relaciones entre actores educativos, entre otros'.

El reclamo de Hargreaves (1998) a los diseñadores de políticas pública sobre el descuido de la dimensión emocional todavía resuena en ámbitos nacionales e internacionales. Sin embargo, la discusión se traduce en un clamor mayor que se podría dirigir hacia los actores mismos de los procesos educativos y de los protagonistas de los currícula (macro-medio y micro) que todavía no consideran esta dimensión en los diseños, prácticas y procesos de mejora para la educación.

\section{Complejidad en la comprensión de las experiencias emocionales}

Una segunda línea teórica que surge de la revisión se orienta a la comprensión misma de las emociones y su incidencia en el ámbito educativo. Este segundo fundamento para la educación emocional se vincula con la concepción compleja y multidimensional de las emociones. Estudios clásicos e investigaciones recientes denotan diversidad de enfoques y concepciones a partir de los varios saberes que abordan las emociones².

La misma denominación conceptual de emociones se transforma a experiencias o episodios. La

$1 \quad$ Véanse: Day y Leitch (2001); Sutton y Wheatley (2003); Hargreaves (2005); Zembylas (2004, 2005a/b, 2007); Van Veen, Sleegers y Van de Ven (2005); Babab (2007); Newberry y Davis (2008); Kelchtermans (2005); 2009; Berger et al. (2009); Sutton y Harper (2009); Frenzel, Goetz, Lüdtke, Pekrun y Sutton (2009); Newberry (2010); Shapiro (2010); Soini, Pyhältö y Pietarinen (2010); Fried (2011); Watkins (2011); Goetz, Lüdtke, Nett, Keller y Lipnevich (2013); Berger, Álamos, Milicic y Alcalay (2014); Finch, Peacock, Lazdowski y Hwang (2015); Goran y Negoescu (2015); Uitto, Jokikokko y Estola (2015); Chen (2016) Raufelder et al. (2016); Uitto, Lutovac, Jokikokko y Kaasila (2018); Mainhard, Oudman, Hornstra, Bosker y Goetz (2018). Véanse: Beatty (2014); Snævarr (2010); Asensio, García, Núñez y Larrosa (2006); Nussbaum (2001); Goldie (2000); Kövecses (2000); Frijda (1986, 2008); Cornelius (1996); Le Doux (1996); Damasio (1996, 2010); Solomon (1993); Lazarus (1991); Denzin (1984). expresión "episodios emocionales" considera la actuación y corregulación de diferentes sistemas dinámicos y componentes en las emociones (Sutton y Wheatley, 2003; Eynde y Turner, 2006), lo que no solo permite ampliar su abordaje investigativo sino también enfocar todos los aspectos de su modelo multicomponente. "Experiencias emocionales" determinadas como estructuras conceptuales almacenadas en la memoria con percepciones, acciones, aspectos cognitivos y afectivos, permiten mapear mejor la riqueza y diversidad de la emoción en representaciones mentales (Barrett, Mesquita, Ochsner y Gross, 2007). Al margen de su estatus ontológico, Beatty (2014) retoma autores y considera las emociones como experiencias unificadas debido a su estructura conceptual o narrativa que indican construcciones de situaciones personales (Goldie, 2000; Roberts, 1988; Shweder, 1994; Solomon, 1993). La denominación de "experiencia emocional" -referida por Schutz y De Cuir (2002) como un proceso cuyo desarrollo tiene un comienzo, un medio y un finalademás de implicar una dimensión cognitiva que requiere autoreflexión e interpretación del contexto y los significados culturales, se determina como una interpretación y evaluación de su estado emocional por parte del individuo.

La comprensión de las experiencias emocionales incluye la diversidad de intentos de clasificación que profundizan su complejidad. La conceptualización eudamonística de Nussbaum considera en las emociones instancias malas y buenas e inclusive su beneficio, tanto para la vida como para el logro de la felicidad, lo cual implica la necesidad de su apropiado cultivo, que determinaría incluso "algo útil en la ira cuando contribuye a levantarnos contra la injusticia" (Modzelewski, 2014, p. 322). Esta concepción contrasta con diversos y complejos intentos de clasificación: emociones básicas o primarias y emociones complejas o secundarias, emociones agradables y desagradables, sentimientos adecuados e inadecuados, emociones pasivas o involuntarias y emo- 
ciones activas o voluntarias, emociones positivas que implican sentimientos agradables y emociones negativas que implican sentimientos desagradables (Brígido, Conde y Bermejo, 2013).

En esta línea teórica de comprensión, más allá de reseñar investigaciones con los diversos conceptos, es sugerente presentar elementos que se presentan en la experiencia emocional y que abren un horizonte para procesos de investigación educativa y para prácticas de educación emocional. Vivas, Gallego y González (2007, pp. 20-21) retoman los conceptos de varios autores y proponen algunos elementos: a) una situación o estímulo que reúne ciertas características, o cierto potencial, para generar tal emoción; b) un sujeto capaz de percibir esa situación, procesarla correctamente y reaccionar ante ella; c) el significado que el sujeto concede a dicha situación, lo que permite etiquetar una emoción, en función del dominio del lenguaje, con términos como alegría, tristeza, enfado, entre otros; d) la experiencia emocional que el sujeto siente ante esa situación; e) la reacción corporal o fisiológica y respuestas involuntarias: cambios en el ritmo cardíaco o respiratorio, aumento de sudoración, cambios en la tensión muscular, sudoración, sequedad en la boca, presión sanguínea; f) la expresión motora-observable: expresiones faciales de alegría, ira, miedo, entre otras; tono y volumen de voz, movimientos del cuerpo, sonrisa, llanto y otros.

Los desarrollos teóricos denotan diversos enfoques para conceptualizar las emociones tanto en procesos de investigación como en prácticas de formación. El reto para fundamentar y desarrollar la educación emocional es no descuidar la complejidad de los referentes teóricos que denotan la dimensión integral del proceso emocional. Prácticas educativas y experiencias emocionales no se podrían sustraer a valorar emociones positivas o negativas, sino que han de fortalecer su desarrollo en los ambientes educativos, con todas sus dimensiones.

\section{Multidimensionalidad de las experiencias emocionales}

Un grupo de investigaciones revisadas marcan el resultado de una tercera línea teórica: la condición multidimensional de las experiencias emocionales o las emociones como procesos multicomponentes (Frijda, 1986; Lazarus, 1991; Planalp, 1999; Sutton y Wheatley, 2003; Zembylas, 2004; Eynde y Turner, 2006; Vivas et al., 2007; Gross, 2014; Beatty, 2014). En este marco complejo, y de acuerdo con las investigaciones revisadas, se podría diferenciar entre múltiples dimensiones o perspectivas para comprender la génesis, desarrollo y expresión de las emociones, por un lado, y la diversidad de escenarios y situaciones que también impactan el origen y desarrollo de las experiencias emocionales, por el otro.

En relación con el primer aspecto, diversas perspectivas de las emociones van desde determinaciones biológicas y genéticas hasta construcciones sociales, pasando por la dimensión individual y cognitiva como respuesta a situaciones particulares (Zembylas, 2004). Por su parte, Sutton y Wheatley (2003, p. 329) refieren el proceso emocional como red de cambios en una variedad de subsistemas (o componentes) del organismo, que incluyen: evaluación, experiencia subjetiva, cambio fisiológico, expresión emocional y tendencias de acción, y Eynde y Turner (2006) consideran los estados y experiencias emocionales compuestos de múltiples componentes que interactúan, y adoptan aquellos componentes identificados por Scherer (2004, p. 138): 1) cognitivo, que es responsable de la evaluación de objetos y eventos; 2) neurofisiológico, que regula la excitación; 3) de expresión motora, que representa la expresión y reacción emocional; 4) motivacional, que prepara y dirige la acción; 5) de sentimiento subjetivo, que monitorea el estado interno y el interacción con el contexto inmediato. En palabras de Gross (2014, p.4), la "naturaleza multifacética" identifica las emociones como fenómenos de todo el cuerpo que implican cambios débilmente acoplados en los dominios de la experiencia subjetiva, el comportamiento y la 
fisiología central y periférica. Más allá de su "estatus ontológico" y como experiencias unificadas, Beatty (2014) considera la necesidad del cuidado de los aspectos conceptuales y metodológicos en el estudio de las emociones, por la dificultad para "capturar" aspectos como su particularidad, su dimensión temporal y cuidar la fidelidad de la experiencia.

El trabajo investigativo de EyndeyTurner (2006) enfocado en la complejidad de las emociones busca desentrañar las interrelaciones de sus procesos cognitivos, neurofisiológicos, motrices, motivacionales y de sentimientos, con base en la teoría de los sistemas, dinámica y componente del proceso emocional. La multidimensionalidad de las emociones se traduce como una "visión integral" que permite mejor comprensión de su naturaleza social y dinámica y de su dimensión de proceso cuyos componentes se regulan mutuamente en el tiempo y dentro de un contexto particular. Esta visión de sistemas dinámica y por componentes implica adecuar instrumentos investigativos para su abordaje en contextos de aprendizaje. De manera particular, el artículo aborda los aspectos relacionados con la interacción entre los procesos emocionales-conativos-cognitivos, con el fin de aclarar las complejas interrelaciones de las emociones, cogniciones, motivaciones y procesos volitivos de los estudiantes. El soporte teórico lo desarrollan Eynde y Turner (2006) con base en los cuatro principios dinámicos de las experiencias emocionales identificados por Mascolo, Harkins y Harakal (2000, p. 127): 1) los estados y experiencias emocionales se componen de procesos de componentes múltiples; 2) las experiencias emocionales surgen a través de la regulación mutua de los sistemas componentes a lo largo del tiempo y dentro de contextos particulares; 3 ) los sistemas de componentes son sensibles al contexto, lo que significa que no solo se ajustan unos a otros, sino también a cambios continuos en el contexto social; 4) las experiencias emocionales se autoorganizan en una serie de patrones o atractores más o menos estables que producen un gran número de variaciones menores.
En contextos de aprendizaje, los resultados investigativos de Eynde y Turner (2006) indican algunos aspectos que pueden coadyuvar a la comprensión de la experiencia emocional en el ámbito educativo, trazan sugerentes desarrollos y plantean desafíos. El paso de un enfoque aislado a uno multidimensional permite el análisis de las dimensiones emocionales de las actividades de los estudiantes en el aula, sin aislarlas de los procesos cognitivos y motivacionales, con la posibilidad de comprender mejor la complejidad de las actividades de aprendizaje en el aula, y sin descuidar aspectos como múltiples metas, aptitudes y características de la personalidad del estudiante. Por otra parte, el enfoque multidimensional de la experiencia emocional permite comprender con mayor profundidad tanto la experiencia misma como el proceso de aprendizaje del estudiante: se podría revelar el modo como este interpreta y valora su aprendizaje, sus creencias y las estructuras de conocimiento subyacentes a sus emociones y se podrían analizar sus valores personales, interpersonales y comunitarios.

Con relación al segundo aspecto sobre la complejidad de la experiencia emocional, es necesario considerar su dimensión social, junto con la diversidad de escenarios y situaciones que ubican las experiencias emocionales más allá de los ámbitos individual y privado y de sus raíces biológicas y cognitivas, que impactan su origen, desarrollo, comprensión y evaluación: aspectos sociales, culturales, institucionales, entornos socioculturales (Mascolo, Harkins y Harakal, 2000; Zembylas, 2004, 2007; Kelchtermans, 2005; Eynde y Turner, 2006; Sutton y Harper, 2009; Du Toit, 2014; Barrios-Tao, 2016). Señalaba Denzin (1984) el paso de la emoción de la esfera individual a la consideración del ámbito social y presenta la comprensión emocional como proceso social e intersubjetivo que requiere, además de ingresar al campo de la experiencia del otro, atender a la vivencia de sus mismas experiencias u otras similares.

Los enfoques de sistemas de componentes dinámicos de Eynde y Turner (2006) apuntan a la 
naturaleza social de las emociones e indican que las experiencias emocionales siempre se sitúan en el contexto social e histórico más amplio e inmediato, lo cual no implica la extrapolación de los procesos biogenéticos, sino su coactuación con los sistemas socioculturales. En palabras de Du Toit (2014, p. 2), sus raíces biológicas se activan mediante el desencadenante contexto cultural. El autor señala que la biología proporciona "mecanismos" emocionales y la cultura codetermina mecanismos desencadenantes y paradigmas en la expresión emocional.

En el marco de sistemas de componentes (Mascolo et al., 2000) asumido por Eynde y Turner (2006), el tercer elemento indica que los sistemas se ajustan a cambios continuos en el entorno o contexto social. Los significados vinculados con situaciones y contextos tienen su base en conocimientos y creencias conectados con las situaciones particulares. De ahí que aspectos del contexto específico escolar impacten los procesos evaluativos de las experiencias emocionales. Eynde y Turner (p. 368) aplican el sistema de componentes al ámbito educativo y afirman que cada emoción se sitúa en su contexto, de acuerdo con cuatro características: 1) las emociones se basan en las interpretaciones cognitivas y las evaluaciones de situaciones específicas de los alumnos;2) los estudiantes construyen interpretaciones y evaluaciones de sus emociones con base en su propio conocimiento y sus creencias y, por tanto, sus interpretaciones están influenciadas y varían según diversos factores: edad, historia personal y cultura del hogar; 3 ) las emociones se contextualizan, porque los individuos crean evaluaciones únicas de los eventos dentro de las situaciones; 4) las emociones son inestables porque tanto las situaciones como la persona inmersa en ellas se desarrollan continuamente.

Los estudios de Zembylas (2004, 2005a/b, 2007), contextualizados en escenarios educativos, discuten, por un lado, el enfoque de la emoción como práctica discursiva con énfasis en el rol constitutivo que juega el lenguaje y, por otro, más allá de disposiciones personales, la construcción social de las emociones de los actores educativos mediante sus relaciones sociales y sistemas de valores provenientes de sus familias y culturas, que también influyen en la expresión y comunicación emocional. Esta dimensión social indica que las experiencias emocionales no solo son estados internos, sino que su referencia debe considerar la vida social, con la consecuencia de las inevitables e inherentes relaciones de poder en la "conversación emocional" (Zembylas, 2004, p. 187). Con base en el pensamiento de Foucault, Zembylas (2005b) analiza la formación emocional de los profesores y el desarrollo de su identidad en relación con el poder institucional. Algunos resultados indican que el gobierno de los profesores se podría ejercer mediante "reglas emocionales" orientadas por las instituciones escolares y legitimadas por medio del ejercicio del poder académico. En consecuencia, se pretendería "normalizar" sus expresiones emocionales con límites relacionados con la disciplina y docilidad. Nobile (2017, p. 28) retoma algunos autores (McWilliam, 1999; Bjerg y Staunæs, 2011) que consideran los discursos pedagógicos orientados al fortalecimiento del "bienestar emocional" como nuevas formas de gobernabilidad en los ámbitos escolares.

En contextos educativos institucionales algunos momentos, eventos y acontecimientos obedecen a la dinámica normal de sus procesos y programas y otros son coyunturales en relación con situaciones sociales o institucionales de los ámbitos nacional o internacional: reformas educativas, presentación a determinadas instancias educativas, renovaciones y acreditaciones institucionales, etc. Estas situaciones no aíslan las experiencias emocionales individuales del devenir institucional y social de estas efemérides. Kelchtermans presenta resultados investigativos sobre las emociones de los profesores en contextos de reformas educativas. Las agendas generadas para estos procesos pueden imponer creencias normativas con el desencadenamiento de intensos sentimientos intensos y la provocación de acciones micropolíticas de resistencia a las condiciones labo- 
rales. Las experiencias emocionales en estos contextos particulares se deben entender en relación con la "vulnerabilidad que constituye una condición estructural del trabajo docente" (2005, p. 95).

En el caso particular del entorno del aula con su desarrollo de las relaciones entre los actores educativos y sus variaciones culturales e individuales, las investigaciones realizadas por educadores indican su impacto en la variabilidad de las emociones de los estudiantes y en sus procesos de aprendizaje3. El escenario escolar se determina como un ambiente sociocultural emocional en donde se generan, configuran y desarrollan variedad de experiencias emocionales que influyen en los procesos educativos (Schutz y De Cuir, 2002) y en donde la apertura de las emociones del ámbito privado y su dimensión psicológica se despliega a las experiencias sociales desarrolladas en el ambiente educativo, con impacto en su organización y caracterización (Zembylas, 2007). Tanto para procesos de investigación educativa como para los de educación emocional es necesaria la consideración de los contextos en donde se vivencian las experiencias emocionales: las aulas, así como las influencias históricas y socioculturales. Asimismo, cuenta la particularidad de la historia de cada ambiente educativo, sus escenarios, aulas, actores educativos y influencias socioculturales: nivel socioeconómico, religiosidad, etnia, género, etc. (Schutz y De Cuir, 2002, 131).

Algunos artículos revisados presentan resultados acerca de las experiencias emocionales de actores educativos en ambientes influenciados por políticas neoliberales (Berg, Huijbens y Gutzen, 2016). Por su lado, Moffatt et al. (2018) contribuyen a mejorar la comprensión de las emociones en contextos universitarios cuando consideran la influencia del neoliberalismo en las prácticas educativas. La lógi-

3 Véanse: Kelchtermans (2005); Zembylas (2007); Frenzel et al. (2009); Sutton y Harper (2009); Berger et al. (2009); Hosotani y Imai-Matsumura (2011); Goetz et al. (2013); Berger, Álamos, Milicic y Alcalay (2014); Becker, Goetz, Morger y Ranellucci (2014); Mainhard et al. (2018). ca del capital y el valor económico, centrales ahora en la vida universitaria, caracterizan una educación neoliberal. El neoliberalismo se materializa en la emoción y su influencia en aspectos como la reorganización de los lugares de trabajo genera emociones configuradas como formas de poder que alinean los cuerpos y causan separación entre ellos. Con la ideología neoliberal surgen nuevas técnicas gerenciales de "gobernanza suave" vinculadas con valores económicos, culturas de auditorías, de clasificación, de eficiencia económica, de gestión documental y de competitividad, en donde los actores educativos se convierten en capital humano o seres economizados, incitados a superar a sus competidores (Berg et al., 2016). Por su parte, Askins y Blazek (2017) confirman la construcción sociocultural de las emociones e identifican ansiedad y deseo en los actores educativos, provocados por factores de competencia y auditoría, lo que tiene un "costo emocional", en una academia neoliberal cada vez más normalizada.

Sutton y Wheatley (2003) consideran los aspectos ambientales, culturales y sociales en la configuración, desarrollo y expresión de las emociones que se reflejan en la teoría de la evaluación, cuya explicación aduce las razones por las cuales un mismo evento externo no configura las mismas emociones en los individuos. De ahí que se puedan evidenciar diferencias individuales en las expresiones emocionales de profesores que responden a los "mismos" comportamientos de determinados estudiantes. En relación con la regulación emocional de los estudiantes, Jiang, Vauras, Volet y Wang (2016) presentan resultados sobre el impacto de factores vinculados con el entorno, como la gestión escolar y la carga de trabajo. Respecto del aprendizaje socioemocional, Berger et al. (2014) indican que no hay que considerarlo exclusivamente como desarrollo de competencias individuales, pues también se deben involucrar variables sociales y estimar la relación del individuo y su contexto.

Procesos de enseñanza-aprendizaje reciben la influencia de variadas experiencias emocionales 
surgidas en escenarios escolares (Schutz y De Cuir, 2002). La discusión acerca de la influencia del contexto sociocultural y de los escenarios y ambientes escolares en los procesos educativos va por doble vía. Por un lado, respecto de la necesidad de considerar el impacto de estos ambientes en la educación emocional y, por otro, acerca del cuidado de los responsables de los procesos educativos para determinar cómo, qué y cuáles aspectos de los escenarios educativos se deberían intervenir para fortalecer la formación de las experiencias emocionales de los actores educativos. La crítica de Nobile (2017) se orienta al peligro de reducir la educación emocional al desarrollo de competencias y al mejoramiento de la inteligencia emocional con instrumentos terapéuticos, extrapolada de situaciones, ambientes y contextos escolares. Las experiencias emocionales no pueden estar deshistorizadas y descontextualizadas, lo cual indica un horizonte de discusión para la educación emocional: considerar factores institucionales y estructurales que impactan las experiencias emocionales de los actores educativos, más allá del desarrollo de rasgos de personalidad orientado a la formación, manipulación y disipación de las denominadas emociones negativas.

\section{El acto educativo: una experiencia emocional}

La cuarta línea teórica se vincula con estudios que identifican el acto educativo como acto o experiencia emocional (Denzin, 1984; Hargreaves, 1998 2001a/b; Zembylas 2007; Sutton y Harper, 2009; García, 2009). En su comprensión de la emoción, Denzin (1984) considera la enseñanza como práctica emocional que supera los aspectos técnico o cognitivo. Además de que todo proceso cognitivo es emocional, la dimensión relacional de quienes intervienen en el acto educativo y las experiencias emocionales generadas y configuradas en ambientes sociales y educativos sustentan la afirmación de que toda acción educativa es una práctica emocional. En consecuencia, "ninguna descripción o explicación so- bre los procesos educativos quedaría completa, sin tomar en consideración los episodios emocionales que intervienen en los procesos formativos cotidianos" (García, 2009, p. 92).

El sugerente referente teórico planteado por Hargreaves es punto de partida fundamental en la relación emociones y enseñanza-aprendizaje, así como en la forma como se investigan y representan las emociones en las relaciones profesor-estudiante: 1) la enseñanza es una práctica emocional; 2) la enseñanza-aprendizaje implica la comprensión emocional; 3) la enseñanza es una forma de labor emocional; 4) las emociones de los profesores son inseparables de sus propósitos morales y de su habilidad para lograr esos propósitos (1998, p. 838). La afirmación "las emociones son el corazón de la enseñanza" conduce al acto de su cuidado, que requiere no solo sensibilidad emocional, sino también un trabajo emocional activo (p. 840).

Para Sutton y Harper (2009) la enseñanza es un esfuerzo emocional apreciable en cada experiencia vivida en el escenario educativo: felicidad cuando se logran objetivos educativos, orgullo por el cumplimiento de las tareas de los estudiantes, frustración ante la dificultad para captar conceptos por parte de los estudiantes, ira por malas conductas, desilusión por falta de esfuerzo. Schuwirth (2013) refiere que las emociones involucradas en el proceso de aprendizaje determinan, por un lado, aquello que el estudiante desea olvidar porque no representan significado alguno y el tiempo dedicado fue considerado inútil, así el material fuera relevante, $y$, por otro lado, lo que el estudiante no quiere olvidar porque despertaron su curiosidad, lo desafiaron, contradijeron y motivaron para buscar mayor información. Las investigaciones neurocientíficas ya indicaban la inclinación de los estudiantes a recordar mejor las cosas cuando se involucran emocionalmente en su aprendizaje: "aulas aburridas es probable que sean aulas ineficientes" (Hall, 2005, p. 23). La discusión acerca de la relación emociones-educación debe trasponer la frontera 
acerca de sus mutuas influencias con investigaciones diagnósticas sobre su impacto, para arribar a propuestas de intervención en escenarios educativos, relaciones entre los actores educativos y el acto mismo de enseñanza-aprendizaje-evaluación, que se configuren como prácticas concretas de educación emocional.

\section{Educación emocional: más allá de inteligencia y competencias socio- emocionales}

Las anteriores líneas teóricas confluyen y sustentan la quinta: la comprensión misma de lo que significaría educación emocional. La educación emocional traza desafíos no solo por la conceptualización sobre la educabilidad de las emociones y los indicadores para su evaluación, sino también por las preocupaciones acerca de la competitividad, el mercadeo y la medición de sus resultados formativos. Estudios pioneros (Gardner, 1995; Goleman, 1996; Damasio, 1996; Mayer y Salovey, 1997, Mayer, Salovey y Caruso, 2000), así como los avances investigativos en neurociencias, desencadenaron procesos de formación emocional centrados en el desarrollo de competencias socioemocionales y de la inteligencia emocional (Bisquerra, 2003; Fernández-Berrocal y Ramos, 2002; Fernández-Berrocal y Ruiz, 2008).

El modelo teórico sobre inteligencia emocional de Mayer y Salovey (1997) integra cuatro niveles, con sus implicaciones: 1) percibir las emociones propias y de los demás: conocer y expresar las emociones; 2) utilizar las emociones: dejar fluir las emociones para que influyan en el pensamiento y ajustar las emociones a las tareas a realizar; 3) comprender las emociones: descubrir el significado de las emociones; conocer qué las causan; qué emociones participan y están involucradas en las situaciones; reconocer el carácter dinámico de las emociones; 4) gestión de emociones: habilidad para regular las emociones propias y su incidencia sobre uno mismo y en los demás; y habilidad para integrar las emociones en el pensamiento.
Gross (2014, 4 ss.) caracteriza las emociones como punto de partida para abordar aspectos de regulación emocional, donde: 1) la emoción se relaciona con su génesis: cuándo ocurre y se asocia al momento en que un individuo atiende y evalúa una situación como relevante para un tipo particular de objetivo (perdurable-transitorio, consciente-inconsciente, simple-complejo) actualmente activo; 2) la emoción es de naturaleza multifacética, involucra todo el cuerpo e implica cambios articulados en los dominios de experiencia subjetiva, el comportamiento y la fisiología central y periférica. Con base en estas características centrales de la emoción, Gross (2014) presenta su modelo modal de la emoción: situación - atención - evaluación - respuesta. La emoción involucra la transacción persona-situación que atrae la atención con un significado para el individuo a la luz de sus objetivos actualmente activos, los cuales generan respuestas multisistémicas flexibles que modifican la transacción en curso persona-situación. El modelo de proceso para regulación emocional de Gross (2014) distingue cinco familias: 1) selección de situación: implica tomar acciones que determinen mayor o menor probabilidad de que se acabe en una situación de la que uno espera que origine emociones deseables (o indeseables); 2) modificación de situación: modificar directamente una situación para alterar su impacto emocional; 3) despliegue de la atención: dirigir la atención sobre una situación para influenciar en las emociones originadas; centrar la atención en otros aspectos de la situación o desviar la atención de la situación; 4) cambio cognitivo: modificar el modo como se evalúa una situación con el fin de alterar su significación emocional; 5) modulación de respuesta: es posterior al proceso generativo de la emoción y se refiere a influenciar directamente los componentes experienciales, conductuales o fisiológicos de la respuesta emocional.

Otro modelo sobre regulación emocional, basado en la psicología social y desarrollado por autores como Metcalfe y Mischel (1999) y Mischel y 
Ayduk (2004), es el Modelo de sistema frí/caliente. El sistema frío se caracteriza por su complejidad, lentitud y contemplación, además de ser cognitivo y emocionalmente neutral. La interconexión de sus redes nodales genera comportamientos racionales, reflexivos y estratégicos. Por su parte, el sistema caliente es activo y permite un procesamiento emocional ágil y simple. El tercer modelo de recursos o fortaleza, desarrollado por autores como Baumeister, Bratslavsky, Muraven y Tice (1998) y Schmeichel y Baumesiter (2004), parte de que cualquier tipo de autorregulación requiere energía o recursos internos y la fuerza autorreguladora es un recurso limitado. De ahí que el agotamiento de estos recursos aumente la probabilidad de falla en la regulación emocional.

Con base en estas teorías y modelos, y en el marco del desarrollo integral de la persona, la educación emocional planteada por Bisquerra es un proceso continuo y permanente orientado a potenciar el desarrollo de competencias emocionales, comprendidas como el "conjunto de conocimientos, capacidades, habilidades y actitudes necesarias para comprender, expresar y regular de forma apropiada los fenómenos emocionales" (2003, p. 22). En este sentido, el objetivo de la educación emocional es el desarrollo de competencias emocionales: conciencia emocional, regulación emocional, autogestión, inteligencia interpersonal, habilidades de vida y bienestar (Bisquerra, 2003; Fernández-Berrocal y Ramos, 2002; Fernández-Berrocal y Ruiz, 2008). Como criterios necesarios en prácticas de educación emocional, Bisquerra considera que la adecuación de los contenidos al nivel de los estudiantes, el favorecimiento de procesos de reflexión sobre las emociones propias y ajenas, y metodologías prácticas.

La revisión realizada abre también la discusión sobre educación emocional, a partir de la concepción misma de la educación, y sobre la necesidad de considerarla más allá de la inteligencia emocional y las competencias socioemocionales, la superación de sus asuntos terapéuticos y la no extrapolación de aspec- tos contextuales en donde se desarrollan las experiencias emocionales (Marina, 2005; Illouz, 2010; Bernal, 2013; Morgan, 2015; Nobile, 2017; Prieto, 2018).

Prieto (2018) analiza la reducción de la educación en emociones a la inteligencia emocional y a teorías e instrumentos de la psicología positiva, que limita no solo al sujeto de la educación en sus posibilidades, su racionalidad y experiencia emocional, sino a la relación educativa, transformada en una relación comunicativa en la cual la acción se sustituye por el discurso. El carácter de la educación centrada en el sujeto y sus posibilidades, cuyo origen brota de la condición del ser humano inacabado, se orienta a su formación permanente relacionada con su propio desarrollo y sus relaciones con los otros y el mundo, y puede recoger, pero no ser sustituido por teorías positivas de la psicología. La propuesta de Marina (2005) busca trascender las habilidades psicológicas entendidas como "único diseño pedagógico" hacia la articulación de múltiples proyectos en un marco más amplio: el de la educación ética. En la elaboración teórica de la inteligencia, para Marina la ética es la "gran creación" de la inteligencia y su punto de llegada está en la neurología (p. 39). En este mismo sentido va la reflexión crítica de González et al. (2013), en el marco amplio de la educación de la afectividad.

Bernal (2013) advierte sobre la posibilidad de diluir y reducir la acción educativa a una actividad terapéutica. Por su parte, Nobile (2017) previene sobre el oscurecimiento de condiciones institucionales, socioeconómicas y culturales de las experiencias emocionales y advierte acerca de la subestimación de su carácter relacional en escenarios educativos. La crítica planteada a la educación por competencias resuena también en propuestas de educación emocional reducidas a la inteligencia emocional, en el sentido de clasificar y provocar en los actores educativos tipos de competencias e incompetencias sociales (Illouz, 2007; 2010) que no contribuyen a la centralidad de los actores en el acto educativo ni a procesos cooperativos y colaborativos de formación integral. 
Más allá del desarrollo de competencias socioemocionales, Morgan (2015) considera dos condiciones que posibilitan la educación de las emociones: normativa y pedagógica. La condición normativa está basada en una educación normativa en sí misma, cuyo resultado es un cambio que mejora la persona. Se educa a la persona, no las emociones y, en consecuencia, se evalúa la vida emocional de la persona o la persona que expresa emociones. La condición pedagógica se fundamenta en la posibilidad del desarrollo parcial de la vida emocional a través del aprendizaje y la enseñanza. La educación emocional se justifica en dos visiones (Romero, 2007): 1) el enfoque de necesidades: con solución de problemáticas relacionadas con déficit en aprendizaje y 2) el enfoque positivo: como vía para cultivar el desarrollo personal y promover el bienestar. En síntesis, como la educación es "una cuestión de transformación de la persona, el aprendizaje debe contribuir a un cambio duradero y profundo en la persona, en lugar de [ser] una habilidad superficial" (Morgan, 2015, p. 292). En este sentido, Marina (2012) considera que "todo aprendizaje cambia el cerebro, pero la educación lo hace de una manera intencionada, dirigida, aprovechando conscientemente las posibilidades que el mismo cerebro proporciona".

\section{Conclusiones}

La multidimensionalidad de las emociones en cuanto a su génesis, expresiones, experiencias y procesos conduce a la complejidad de su abordaje en los escenarios educativos y desafía las investigaciones y estudios en las áreas de las ciencias sociales y humanas, así como las propuestas de educación emocional. Esta se encamina a fortalecer sus fundamentos, enfoques y prácticas con una comprensión y orientación de la educación que considere la centralidad y complejidad del ser humano. Más allá de la pregunta por la educabilidad de las emociones, en momentos determinados, con técnicas específicas y desarrollos de habilidades psicológicas, la mirada se debe orientar hacia la persona que se educa y hacia sus experiencias emocionales, cuya comprensión, configuración y refiguración se conducen por el aprendizaje ampliado para toda la vida, en todos los momentos. Con Vázquez y Manassero se puede concluir que la educación emocional "debería facilitar el desarrollo emocional de las personas a través de las enseñanzas dirigidas a aprender la sabiduría de los sentimientos" (2007a, p. 250).

La complejidad de las emociones, en lo que respecta a sus dimensiones y a los escenarios que influyen en su génesis y expresión, indica la necesidad de no extrapolar a la educación emocional factores como el ambiente escolar y las influencias provenientes de sus entornos socioculturales. Los aspectos estructurales e institucionales no solo contribuyen a la mejor comprensión de las experiencias emocionales, sino también a su intervención formativa para fortalecer su adecuada expresión. La consideración de los factores ambientales de los escenarios educativos permitiría fortalecer una "prioridad educativa significativa": crear condiciones para una mejor comprensión emocional entre los actores educativos (Hargreaves, 1998, p. 840).

Varias teorías educativas han estudiado la relación entre los procesos cognitivos y los factores individuales, sociales, culturales, biológicos, psicológicos y tal estudio se enriquece ahora con investigaciones neurocientíficas y otros estudios contextualizados en ambientes educativos. Los inicios del siglo XXI trazan un horizonte que se podría determinar todavía como un desafío tanto para los actores de los procesos integrales en educación como para las personas comprometidas en aspectos institucionales, quienes tienen la misión de formular políticas públicas. A unos y otros ya no se les admite la discusión sobre la necesidad de considerar las experiencias emocionales como un factor fundamental en las acciones educativas, el desafío para todos es la conciencia, comprensión y formación acerca de las emociones. El llamado de Hargreaves (1998, p. 851) adquiere mayor relevancia cuanto más avanzan los estudios, experiencias e investigaciones: "mejor comprensión emocional, menos emociones espurias". 
La responsabilidad de la educación emocional debe trascender la relación profesor-estudiante, el desarrollo de habilidades o competencias socioemocionales y el supuesto fortalecimiento de una inteligencia emocional para orientarse al desarrollo integral en la formación, expresión y comunicación de las experiencias emocionales de los actores educativos, de manera que se integre -en las agendas de todos los responsables institucionales en todos los nivelesel cuidado de los ambientes que permitan desarrollar mejores episodios emocionales en escenarios y espacios temporales adecuados. Los actos educativos, que son actos relacionales y emocionales, exigen cuidado para un desarrollo integral formativo.

\section{Referencias}

Ainley, M. (2006). Connecting with learning: Motivation, affect, and cognition. Educational Psychology Review, 18, 391-405. DOI: https://psy cnet.apa.org/doi/10.1007/s10648-006-9033-0

Asensio, J. M., García Carrasco, J., Núñez Cubero, L.y Larrosa, J. (coords.) (2006). La vida emocional. Las emociones y la formación de la identidad humana. Barcelona: Ariel.

Askins, K.y Blazek, M. (2017). Feeling our way: academia, emotions and a politics of care. Social and Cultural Geography, 18(8), 1086-1105. DOI: https://doi.org/10.1080/14649365.2016.1240224

Barrios-Tao, H. (2016). Neurociencias, educación y entorno sociocultural. Educación y Educadores, 19(3), 395-415. DOI: https://doi.org/10.5294/edu.2016.19.3.5

Baumeister, R. F., Bratslavsky, E., Muraven, M. y Tice, D. M. (1998). Ego depletion: Is the active self a limited resource? Journal of Personality and Social Psychology, 74, 1252-1265. DOl: https://doi.org/10.1037//00223514.74 .5 .1252

Blakemore, S. J. y Frith, U. (2000). Report on the implications of recent developments in neuroscience for research on teaching and learning. A consultation paper commissioned by the Teaching and Learning Research Programme, ESRC.

Blakemore, S. J. y Frith, U. (2005). The learning brain: Lessons for education. Oxford: Blackwell.

Babab, E. (2007). Teachers' nonverbal behaviors and its effects on students. En Perry, R.y Smart, J. C. (eds.), The scholarship of teaching and learning in higher education: An evidence-based perspective (pp. 201-261). Nueva York: Springer.

Barrett, L. F., Mesquita, B., Ochsner, K. y Gross, J. (2007). The experience of emotion. Annual Review of Psychology 58, 373-403. DOI: http://doi.org/10.1146/annurev.psy ch.58.110405.085709

Beatty, A. (2014). Anthropology and emotion. Journal of the Royal Anthropological Institute, 20, 545-563. DOI: https://doi.org/10.1111/1467-9655.12114

Becker, E. S., Goetz, T., Morger, V. y Ranellucci, J. (2014). The importance of teachers' emotions and instructional behavior for their students' emotions - An experience sampling analysis. Teaching and Teacher Education 43, 15-26. DOI: https://doi.org/10.1016/j.tate.2014.05.002 
ISSN 0123-1294 | e-ISSN 2027-5358 | Educ.Educ. Vol. 22. No.3 | Septiembre-diciembre de 2019 | pp. 487-509.

Universidad de La Sabana | Facultad de Educación

Berg, L., Huijbens, E. y Gutzen Larson, H. (2016). Producing anxiety in the neoliberal university. The Canadian Geographer, 6o(2), 168-180. DOI: https://doi.org/10.1111/cag.12261

Berger, C. et al. (2009). Bienestar socioemocional en contextos escolares: la percepción de estudiantes chilenos. Estudios sobre Educación, 17, 21-43. Recuperado de https://www.unav.edu/publicaciones/revistas/index.php/estudios-sobre-educacion/article/view/22422

Berger, C., Álamos, P., Milicic, N. y Alcalay, L. (2014). Rendimiento académico y las dimensiones personal y contextual del aprendizaje socioemocional: Evidencias de su asociación en estudiantes chilenos. Universitas Psychologica, 13(2), 627-638. DOI: https://doi.org/10.11144/Javeriana.UPSY13-2.radp

Bernal, A. (2013). La relación educativa, identidad y emociones. En Flamarique, L. y D’Olivira-Martins, M. (eds.), Emociones y estilos de vida (pp. 75-99). Madrid: Biblioteca Nueva.

Bisquerra, R. (2003). Educación emocional y competencias básicas para la vida. Revista de Investigación Educativa, 21(1), 7-43. Recuperado de https://revistas.um.es/rie/article/view/99071

Bisquerra, R. y Pérez, N. (2012). Educación emocional: Estrategias para su puesta en práctica. Avances en Supervisión Educativa, 16. Recuperado de https://avances.adide.org/index.php/ase/article/view/5O2

Bjerg, H. y Staunaes, D. (2011). Self-management through shame. Uniting governmentality studies and the 'affective turn'. Ephemera. Theory y Politics in Organization, 11(2), 138-156. Recuperado de http://www.ephemerajournal.org/contribution/self-management-through-shame-\%E2\%80\%93-uniting-governmentalitystudies-and-affective-turn

Brígido, M., Conde, M. C. y Bermejo, M. L. (2013). Relación entre el aprendizaje y la enseñanza de las ciencias en la formación inicial del profesorado de primaria. En Mellado, V., Blanco, L. J., Borrachero, A. B.y Cárdenas, J. A. (eds.), Las emociones en la enseñanza y el aprendizaje de las ciencias y las matemáticas (pp. 329-350). Badajoz, España: Deprofe.

Chen, J. (2016). Understanding teacher emotions: The development of a teacher emotion inventory. Teaching and Teacher Education, 55, 68-77. DOI: https://doi.org/10.1016/j.tate.2016.01.001

Citri, A. y Malenka, R. (2008). Synaptic plasticity: Multiple forms, functions, and mechanisms. Neuropsychopharmacology, 33, 18-41. DOI: https://doi.org/10.1038/sj.npp.1301559

Coll, M. (2011). Plasticidad cerebral y experiencia: fundamentos neurobiológicos de la educación. Ponencia XII Congreso Internacional de Teoría de la Educación, Universidad de Barcelona. Recuperado de http:// www.cite2011.com/Ponencias/MColl.pdf

Cornelius, R. R. (1996). The science of emotion: Research and tradition in the psychology of emotion. Nueva Jersey: Prentice-Hall.

Damasio, A. (1996). El error de Descartes. La razón de las emociones. Santiago de Chile: Andrés Bello.

Damasio, A. (2010). Y el cerebro créo al hombre. ¿Cómo pudo el cerebro generar emociones, sentimientos, ideas y el yo? Barcelona: Destino. 
Day, Ch. y Leitch, R. (2001). Teachers' and teacher educators' lives: the role of emotion. Teaching and Teacher Education, 17 (4), 403-415. DOI: https://doi.org/10.1016/S0742-051X(01)00003-8

Denzin, N. K. (1984). On understanding emotion. Michigan: Jossey-Bass.

De Jong, T. (ed.) (2008). Explorations in learning and the brain: On the potential of cognitive neuroscience for educational science. La Haya: Netherlands Organisation for Scientific Research.

Dueñas, M. (2002). Importancia de la inteligencia emocional: un nuevo reto para la orientación educativa. Educación, 21(5). DOI: https://doi.org/10.5944/educxx1.5.1.384

Du Toit, C. W. (2014). Emotion and the affective turn: Towards an integration of cognition and affect in real life experience. HTS Toelogiese Studies/Theological Studies, 70(1), 1-9. DOI: https://hts.org.za/index.php/ HTS/article/view/2692

Eynde, P. O. y Turner J. E. (2006). Focusing on the complexity of emotion issues in academic learning: A dynamical component systems approach. Educational Psychology Review 18, 361-376. DOI: https://doi. org/10.1007/s10648-006-9031-2

Fernández-Berrocal, P. y Ramos, N. (2002). Corazón y razón. En Fernández-Berrocal, P. y Ramos, N. (eds.), Corazones inteligentes (pp. 17-34). Barcelona: Kairós.

Fernández-Berrocal, P. y Ruiz, D. (2008). La inteligencia emocional en la educación. Revista Electrónica de Investigación Psicoeducativa, 15, 6(2), 421-436. Recuperado de http://ojs.ual.es/ojs/index.php/EJREP/article/ view/1289/1363

Finch, D., Peacock, M., Lazdowski, D. y Hwang, M. (2015). Managing emotions: A case study exploring the relationship between experiential learning, emotions, and student performance. The International Journal of Management Education 13, 23-36. DOI: https://doi.org/10.1016/j.jme.2014.12.001

Fontinha, B. et al. (2009). Adenosine A2A receptor modulation of hippocampal CA3-CA1 synapse plasticity during associative learning in behaving mice. Neuropsychopharmacology, 34, 1865-1874. DOI: https://doi. org/10.1038/npp.2009.8

Frenzel, A. C., Goetz, T., Lüdtke, O., Pekrun, R.y Sutton, R. E. (2009). Emotional transmission in the classroom: Exploring the relationship between teacher and student enjoyment. Journal of Educational Psychology, 101(3), 705-716. DOI: https://doi.org/10.1037/aoo14695

Fried, L. (2011). Teaching teachers about emotion regulation in the classroom. Australian Journal of Teacher Education, 36(3). Disponible en: http://dx.doi.org/10.14221/ajte.2011v36n3.1

Frijda, N. (1986). The emotions. Cambridge: Cambridge University Press.

Frijda, N. (2008). The psychologists' point of view. En Lewis, M., Haviland-Jones, J. M. y Feldman Barrett, L. (eds.), Handbook of Emotions (pp. 68-87). Nueva York: Guilford Press.

García, E. (2008). Neuropsicología y educación. De las neuronas espejo a la teoría de la mente. Revista de Psicología y Educación, 1(3), 69-90. Recuperado de http://www.revistadepsicologiayeducacion.es/pdf/27.pdf 
ISSN 0123-1294 | e-ISSN 2027-5358 | Educ.Educ. Vol. 22. No.3 | Septiembre-diciembre de 2019 | pp. 487-509.

Universidad de La Sabana | Facultad de Educación

García, J. (2009). Educación, cerebro y emoción. Aula 15, 91-115. Recuperado de http://revistas.usal.es/index.php/O214-3402/article/viewFile/8945/10445

García, J. A. (2012). La educación emocional, su importancia en el proceso de aprendizaje. Revista Educación, 36(1), 97-109. Recuperado de https://revistas.ucr.ac.cr/index.php/educacion/article/view/455/9906

Gardner, H. (1995). Inteligencias múltiples. La teoría en la práctica. Barcelona: Paidós.

Goetz, T., Cronjaeger, H., Frenzel, A. C., Ludtke, O. y Hall, N. C. (2010). Academic self-concept and emotion relations: Domain specificity and age effects. Contemporary Educational Psychology, 35 (1), 44-58. DOI: https:// doi.org/10.1016/j.cedpsy ch.2009.10.001

Goetz, T., Lüdtke, O., Nett, U. E., Keller, M. M. y Lipnevich, A. A. (2013). Characteristics of teaching and student emotions in the classroom: Investigating differences across domains. Contemporary Educational Psychology, 38(4), 383-394. DOI: https://doi.org/10.1016/j.cedpsy ch.2013.08.001

Goldie, P. (2000). The emotions: A philosophical exploration. Nueva York: Oxford University Press.

Goleman, D. (1996). La inteligencia emocional. Buenos Aires: Javier Vergara.

González, J. (2010). El victimismo elevado a arte. La nueva cultura emocional. Multidisciplina, 7, 15-27. Recuperado de http://quazar.acatlan.unam.mx/multidisciplina/103/

González, P. et al. (2013). Educar las emociones para promover la formación ética. Persona y Bioética, 17(1), 28-48. DOI: https://doi.org/10.5294/pebi.2013.17.1.2

Goran, L. y Negoescu, G. (2015). Emotions at work. The management of emotions in the act of teaching. Procedia - Social and Behavioral Sciences, 180, 1605-1611. DOI: https://doi.org/10.1016/j.sbspro.2015.02.314

Goswami, U. (2004). Neuroscience and education. British Journal of Educational Psychology, 74, 1-14. DOI: https://doi.org/10.1348/000709904322848798

Goswami, U. (2006). Neuroscience and education: From research to practice? Nature Reviews Neuroscience, 7, 406-413. DOI: https://doi.org/10.1038/nrn1907

Gross, J. J. (2014). Emotion regulation: Conceptual and empirical foundations. En Gross, J. J. (ed.), Handbook of emotion regulation (pp. 3-20). Nueva York: Guilford.

Gruart, A. (2014). The role of Neurosciences in Education... and viceversa. International Journal of Educational Psychology, 3(1), 21-48. DOI: https://dx.doi.org/10.4471/ijep.2014.02

Hall, J. (2005). Neuroscience and education. A review of the contribution of brain science to teaching and learning, SCRE Research Report No 121, University of Glasgow.

Hargreaves, A. (1998). The emotional practice of teaching. Teaching and Teacher Education, 14, (8) 835854. DOI: https://doi.org/10.1016/S0742-051X(98)00025-0

Hargreaves, A. (2001a). Emotional geographies of teaching. Teachers College Record, 103(6), 1056-1080. Recuperado de https://www.tcrecord.org/library/abstract.asp?contentid=10838 
Hargreaves, A. (2001b). The emotional geographies of teachers' relations with colleagues. International Journal of Educational Research 35(5), 503-527. DOI: https://doi.org/10.1016/S0883-0355(02)00006-X

Hargreaves, A. (2005). Educational change takes ages: Life, career and generational factors in teachers' emotional responses to educational change. Teaching and Teacher Education, 21(8), 967-983. DOI: https://doi. org/10.1016/j.tate.2005.06.007

Hillman, C. H., Erickson, K. L. y Kramer, A. F. (2008). Be smart, exercise your heart: Exercise effects on brain and cognition. Nature Reviews Neuroscience, 9, 58-65. DOI: https://doi.org/10.1038/nrn2298

Hosotani, R. y Imai-Matsumura, K. (2011). Emotional experience, expression, and regulation of high-quality Japanese elementary school teachers. Teaching and Teacher Education, 27(6), 1039-1048. DOI: https://doi. org/10.1016/j.tate.2011.03.010

Illouz, E. (2007). Las intimidades congeladas. Las emociones en el capitalismo. Madrid: Katz.

Illouz, E. (2010). La salvación del alma moderna. Terapia, emociones y la cultura de la autoayuda. Buenos Aires: Katz

Jankowski, K. F. y Takahashi, H. (2014). Cognitive neuroscience of social emotions and implications for psy chopathology: examining embarrassment, guilt, envy, and schadenfreude. Psychiatry and Clinical Neurosciences, 68(5), 319-336. DOI. https://doi.org/10.1111/pcn.12182

Jiang, J., Vauras, M., Volet, S. y Wang, Y. (2016). Teachers' emotions and emotion regulation strategies: Self- and students' perceptions. Teaching and Teacher Education, 54, 22-31. DOI: https://doi.org/10.1016/j. tate.2015.11.008

Kelchtermans, G. (2005). Teachers' emotions in educational reforms: Self-understanding, vulnerable commitment and micropolitical literacy. Teaching and Teacher Education, 21(8), 995-1006. DOI: https://doi. org/10.1016/j.tate.2005.06.009

Kelchtermans, G. (2009). Who I am in how I teach is the message: self-understanding, vulnerability and reflection. Teachers and Teaching: Theory and Practice, 15(2), 257-272. DOI: https://doi. org/10.1080/13540600902875332

Kövecses, Z. (2000). Metaphor and emotion. Cambridge: Cambridge University Press.

Lazarus, R. S. (1991). Emotion and adaptation. Nueva York: Oxford University Press.

Le Doux, J. (1996). The emotional brain. Nueva York: Simon y Schuster.

Letelier, L. M., Manríquez, J. y Rada, G. (2005). Revisiones sistemáticas y metanálisis: ¿Son la mejor evidencia? Revista Médica de Chile, 133(2), 246-249. Recuperado de http://publicacionesmedicina.uc.cl/Boletin/20052/RevisionesSistematicas.pdf

Mainhard, T., Oudman, S., Hornstra, L., Bosker, R. J. y Goetz, T. (2018). Student emotions in class: The relative importance of teachers and their interpersonal relations with students. Learning and Instruction, 53, 109-119. DOI: https://doi.org/10.1016/j.learninstruc.2017.07.011 
ISSN 0123-1294 | e-ISSN 2027-5358 | Educ.Educ. Vol. 22. No.3 | Septiembre-diciembre de 2019 | pp. 487-509.

Universidad de La Sabana | Facultad de Educación

Marina, J. A. (2005). Precisiones sobre la Educación Emocional. Revista Interuniversitaria de Formación del Profesorado, 19(3), 27-43. Recuperado de https://dialnet.unirioja.es/servlet/articulo?codigo=2126751

Marina, J. A. (2012). El diálogo entre neurociencia y educación. Participación Educativa, 1, 7-14. Recuperado de. https://es.calameo.com/read/00105541934b841014d3b

Martin, R. E. y Ochsner, K. N. (2016). The neuroscience of emotion regulation development: implications for education. Current Opinion in Behavioral Sciences, 10, 142-148. DOI: https://doi.org/10.1016/j.cobeha.2016.06.006

Mascolo, M.F., Harkins, D. y Harakal, T. (2000). The dynamic construction of emotions: Varieties of anger. En Lewis, M. D. y Granic, I. (eds.), Emotion, development, and self-organization: Dynamic systems approaches to emotional development (pp. 125-152). Cambridge: Cambridge University Press.

Mayer, J. D. y Salovey, P. (1997). What is emotional intelligence? En Salovey, P. y Sluyter, D. (eds), Emotional development and emotional intelligence: Implications for educators (pp. 3-31). Nueva York: Basic Books.

Mayer, J. D., Salovey, P. y Caruso, D. (2000). Emotional intelligence. En Sternberg, R. J. (2000), Handbook of Intelligence (pp. 396-421). Nueva York: Cambridge University Press.

Mayo, E. (1977). Problemas sociales de una civilización industrial. Buenos Aires: Nueva Visión.

Mcwilliam, E. (1999). Pedagogical pleasures. Nueva York: Peter Lang.

Metcalfe, J. y Mischel, W. (1999). A hot/cool-system analysis of delay of gratification: Dynamics of willpower. Psychological Review, 106, 3-19. DOI: https://doi.org/10.1037/0033-295X.106.1.3

Mischel, W. y Ayduk, O. (2004). Willpower in a cognitive-affective processing system: The dynamics of delay or gratification. En Vohs, K. D. y Baumeister, R. F. (eds.), Handbook of self-regulation: Research, theory, and applications (pp. 99-129). Nueva York: Guilford Press.

Modzelewski, H. (2014). Autorreflexión y educación de las emociones para la democracia. Entrevista a Martha Nussbaum. Areté. Revista de Filosofía, 26(2), 315-333. Recuperado de http://revistas.pucp.edu.pe/index. php/arete/article/view/11111

Moffatt, K. et al. (2018). Worry about professional education: Emotions and affect in the context of neoliberal change in postsecondary education. Emotion, Space and Society 26, 9-15. DOI: https://doi.org/10.1016/j. emospa.2017.10.006

Morgan, J. (2015). Emptiness and the Education of the Emotions. Educational Philosophy and Theory, 47(3), 291-304. DOI: https://doi.org/10.1080/00131857.2013.860873

Newberry, M. y Davis, H. A. (2008). The role of elementary teachers' conceptions of closeness to students on their differential behaviour in the classroom. Teaching and Teacher Education, 24(8), 1965-1985. DOI: https:// doi.org/10.1016/j.tate.2008.02.015

Newberry, M. (2010). Identified phases in the building and maintaining of positive teacher? student relationships. Teaching and Teacher Education, 26(8), 1695-1703. DOI: https://doi.org/10.1016/j.tate.2010.06.022 
Nobile, M. (2017). Sobre la 'educación emocional': subjetividad y psicologización en la modernidad tardía. Digithum, 20, 22-33. DOI: https://doi.org/10.7238/d.voi20.3089

Nussbaum, M. (2001). Upheavals of thought: the intelligence of emotions. Cambridge: Cambridge University Press.

OCDE (2007). La comprensión del cerebro. El nacimiento de una ciencia del aprendizaje. Santiago: Universidad Católica Silva Henríquez.

Pekrun, R., Goetz, T., Titz, W. y Perry, R. P. (2002). Academic emotions in students' self-regulated learning and achievement: A program of quantitative and qualitative research. Educational Psychologist, 37(2), 91-106. DOI: https://doi.org/10.1207/S15326985EP3702_4

Pekrun, R. (2005). Progress and open problems in educational emotion research. Learning and Instruction, 15(5), 497-506. DOI: https://doi.org/10.1016/j.learninstruc.2005.07.014

Planalp, S. (1999). Communicating Emotion: Social moral and cultural processes. Cambridge: Cambridge University Press.

Popay, J. et al. (2006). Guidance on the conduct of narrative synthesis in systematic reviews. A product from the ESRC Methods Programme. Londres: ESRC.

Prieto Egido, M. (2018). La psicologización de la educación: Implicaciones pedagógicas de la inteligencia emocional y la psicología positiva. Educación XX1, 21(1),303-320. DOI: https://doi.org/10.5944/educxx1.20200

Raufelder, D. et al. (2016). Students' perception of good and bad teachers. Results of a qualitative thematic analysis with German adolescents. International Journal of Educational Research, 75, 31-44. DOI: https://doi. org/10.1016/j.jer.2015.11.004

Roberts, R. C. (1988). What an emotion is: a sketch. The Philosophical Review, 97, 183-209. Recuperado de https://www.jstor.org/stable/2185261?origin=crossref\&seq=1\#page_scan_tab_contents

Romero, C. (2007). ¿Educar las emociones?: Paradigmas científicos y propuestas pedagógicas. Cuestiones Pedagógicas, 18, 105-119. Recuperado de http://institucional.us.es/revistas/cuestiones/18/07\%20educar\%20 las\%2oemociones.pdf

Scherer, K. R. (2004). Feelings integrate the central representation of appraisal-driven response organization in emotion. En Manstead, A. S. R., Frijda, N. y Fischer, A. (eds.), Feelings and emotions: The Amsterdam symposium (pp. 136-157). Cambridge: Cambridge University Press.

Schmeichel, B. J.y Baumesiter, R. F. (2004). Self-regulatory strength. En Vohs, K. D. y Baumeister, R. F. (eds.), Handbook of self-regulation: Research, theory, and applications (pp. 84-98). Nueva York: Guilford Press.

Schutz, P. A. y De Cuir, J.T. (2002). Inquiry on emotions in education. Educational Psychologist, 37(2), 125-134. DOI: https://doi.org/10.1207/S15326985EP3702_7

Schuwirth, L. (2013). 'Emotions in learning' is more than merely 'learning of emotions'. Medical Education, 47(1), 14-15. DOI: https://doi.org/10.1111/medu.12078 
ISSN 0123-1294 | e-ISSN 2027-5358 | Educ.Educ. Vol. 22. No.3 | Septiembre-diciembre de 2019 | pp. 487-509.

Universidad de La Sabana | Facultad de Educación

Shapiro, S. (2010). Revisiting the teachers' lounge: Reflections on emotional experience and teacher identity. Teaching and Teacher Education, 26(3), 616-621. DOI: https://doi.org/10.1016/j.tate.2009.09.009

Shweder, R. A. (1994) “You're not sick, you're just in love": emotion as an interpretive system. En Ekman, P.y Davidson, R. J. (eds.), The nature of emotion: fundamental questions (pp. 23-44). Oxford: Oxford University Press.

Singh, S. y Mishra, R. C. (2011). Emotion regulation strategies and their implications for well-being. Social Science International, 27(2), 179-198. Recuperado de https://www.printspublications.com/carts

Snævarr, S. (2010). Metaphors, narratives, emotions. Their interplay and impact. Ámsterdam: Rodopi.

Soini, T., Pyhältö, K. y Pietarinen, J. (2010). Pedagogical well-being: Reflecting learning and well-being in teachers' work. Teachers and Teaching. Theory and Practice, 16(6), 735-751. DOI: https://doi.org/10.1080/135406 02.2010.517690

Solomon, R. C. (1993). The Passions Emotions and the Meaning of Life. Indianapolis: Hackett.

Sutton,R.E.yWheatley,K.F.(2003).Teachers'emotionsand teaching:A review of theliteratureand directions for future research. Educational Psychology Review, 15(4), 327-358. DOI: https://doi.org/10.1023/A:1026131715856

Sutton, R. E. y Harper, E. (2009). Teachers' emotion regulation. En Saha, L. J. y Dworkin, A. G. (eds.), International Handbook of Research on Teachers and Teaching (pp. 389-401). Nueva York: Springer.

Uitto, M., Lutovac, S., Jokikokko, K. y Kaasila, R. (2018). Recalling life-changing teachers: Positive memories of teacher-student relationships and the emotions involved. International Journal of Educational Research, 87, 47-56. DOI: https://doi.org/10.1016/j.ijer.2017.11.004

Uitto, M., Jokikokko, K. y Estola, E. (2015). Virtual special issue on teachers and emotions in Teaching and teacher education (TATE) in 1985-2014. Teaching and Teacher Education, 50, 124-135. DOI: https://doi. org/10.1016/j.tate.2015.05.008

Van Veen, K., Sleegers, P. y Van de Ven, P-H. (2005). One teacher's identity, emotions, and commitment to change: A case study into the cognitive-affective processes of a secondary school teacher in the context of reforms. Teaching and Teacher Education, 21(8) 917-934. DOI: https://doi.org/10.1016/j.tate.2005.06.004

Vázquez, A. y Manassero, M. A. (2007a). En defensa de las actitudes y emociones en la educación científica (I): evidencias y argumentos generales. Revista Eureka sobre Enseñanza y Divulgación de las Ciencias, 4(2), 247271. DOI: https://dx.doi.org/10.25267/Rev_Eureka_ensen_divulg_cienc.2007.v4.i2.03

Vázquez, A. y Manassero, M. A. (2007b). En defensa de las actitudes y emociones en la educación científica (II): evidencias empíricas derivadas de la investigación. Revista Eureka sobre Enseñanza y Divulgación de las Ciencias, 4(3), 417-441. DOI: https://dx.doi.org/10.25267/Rev_Eureka_ensen_divulg_cienc.2007.v4.i3.03

Vivas, M., Gallego, D. y González, B. (2007). Educar las emociones. Mérida: Producciones Editoriales.

Watkins, M. (2011). Teachers' tears and the affective geography of the classroom. Emotion, Space and Society, 4,137-143. DOI: https://doi.org/10.1016/j.emospa.2010.03.001 
Yiend, J. (2010). The effects of emotion on attention: a review of attentional processing of emotional information. Cognition and Emotion, 24(1), 3-47. DOI: https://doi.org/10.1080/02699930903205698

Zembylas, M. (2004). The emotional characteristics of teaching: an ethnographic study of one teacher. Teaching and Teacher Education, 20(2), 185-201. DOI: https://doi.org/10.1016/j.tate.2003.09.008

Zembylas M. (2005a). Discursive practices, genealogies, and emotional rules: A poststructuralist view on emotion and identity in teaching. Teaching and Teacher Education, 21(8), 935-948. DOI:https://doi.org/10.1016/j. tate.2005.06.005

Zembylas, M. (2005b). Teaching with emotion: A postmodern enactment. Charlotte, NC: Information Age Publishing.

Zembylas, M. (2007). Theory and methodology in researching emotions in education. International Journal of Research y Method in Education, 30(1), 57-72. DOI: https://doi.org/10.1080/17437270701207785 
